\title{
Changing a Culture with Patient Safety Walkarounds
}

\author{
$\overline{\text { Guna Budrevics and Catherine O’Neill }}$
}

\section{BACKGROUND}

It is evident within Canada and abroad that healthcare is a high-risk industry not unlike aviation, nuclear energy and offshore oil drilling. The results from the Canadian Adverse Events Study (Baker et al. 2004) found an adjusted adverse event rate of $7.5 \%$ in Canadian hospitals. The consequences of these adverse events include prolonged length of stay, varying degrees of injury, and in some instances death. These injuries and deaths are not attributable to the patients' diagnoses but are, in fact, a result of the care provided to them by healthcare practitioners. This study is a part of a growing body of literature that has provided the momentum in many healthcare organizations, including Sunnybrook \& Women's College Health Science Centre ( $\& \& W)$, to ensure patient safety is a priority.

$\mathrm{S} \& \mathrm{~W}$ is dedicated to becoming the safest hospital in Canada. In order to achieve this goal, we must create a culture of safety, which requires significant organizational change. We have implemented several initiatives which have created the impetus for this culture change. One of the most influential initiatives is a patient safety walkarounds program. This well-tested concept has been used in many other healthcare organizations in the United States and in other nonhealthcare-related industries. We have created a model that is an adaptation of the original WalkRounds ${ }^{\mathrm{TM}}$ framework created by Dr. Allan Frankel, (2003) Director of Patient Safety for Partners HealthCare System in Boston, Massachusetts.
Representatives from the Patient Safety Service at the Partners HealthCare System in Boston were instrumental in our understanding the effectiveness of the walkarounds model. By sharing their experiences and openly discussing the merits and development of WalkRounds ${ }^{\mathrm{TM}}$, we were able to construct a similar model that was applicable to a Canadian healthcare delivery system. Some of the adaptations that we made included: a revision of the documentation and communication tools; a modified list of questions to guide the dialogue; inviting managers and directors to hear the walkaround session; and the creation of a handbook to orient the senior leadership team.

Patient safety walkarounds provide any healthcare organization a unique opportunity to facilitate the foundation of a safe culture. Walkarounds in their very essence connect frontline staff with senior leaders in an open dialogue concerning patient safety. This interaction allows frontline staff to share their safety concerns with senior leaders, as well as creating a forum to promote the awareness of patient safety. Conversely, it is an opportunity for senior leaders to demonstrate their commitment by hearing concerns and removing the barriers to safe care.

\section{Roles And ResponsibILITIES - SENIOR LEADERSHIP}

The primary focus of walkarounds is to promote patient safety, yet in order for the mechanics to work, trust and a cultural 
acceptance of disclosure and accountability is critical. In order to promote disclosure and accountability, the S\&W Board of Directors passed two fundamental policies: a Disclosure of Adverse Events policy, and an Accountability for Patient Safety policy. Both policies are the cornerstones of our overall patient safety program and have paved the way for other operational initiatives, such as walkarounds, to be considered and implemented. The role and support of the senior leaders is critical in the success of walkarounds, as they must demonstrate these new behaviors of disclosure and accountability by addressing unitspecific issues in a proactive and responsive manner.

The senior leaders are assigned to units on a rotational schedule. Once each week a senior leader conducts walkarounds in a patient care area. The walkarounds are conducted in a meeting room adjacent to the patient care unit or in the patient care area itself. After introductions and a brief outline of the process, the senior leader guides the dialogue with the use of questions that have been pre-circulated to the staff. These questions stimulate the discussion and encourage participants to share their concerns about patient safety as well as their suggestions for improvement. Throughout the discussion it is likely that the conversation may divert to nonsafety matters, and so the senior leader must focus strictly on patient safety and refrain from discussing competing priorities such as budgets, staffing and other operational crises.

Once the walkarounds are completed, a list of all the comments and issues raised are sent to the manager and staff. From this list they are asked to select the three issues they feel have the most significant impact on their ability to provide safe care. These issues are then delegated to the senior leader who conducted the walkarounds and is responsible for taking the necessary actions to resolve them. Throughout the resolution phase, the senior leader is expected to provide timely feedback on actions. Some issues make take weeks (and perhaps months) to resolve; therefore direct communication to the managers and frontline staff on the progress of the priority issues demonstrates commitment to the initiative. Successful resolution of identified issues shows that patient safety is a high priority for the organization and assists in building a trust between frontline staff and senior leaders.

\section{PREPARATION/EXPECTATIONS}

To prepare for walkarounds, we took several steps to ensure that participants felt prepared and knew what to expect. Articulating the true intent of these rounds at the outset was critical, as there was a risk that they may be viewed as an inspection of the unit versus a nonthreatening discussion about patient safety. We met with the managers of the patient care areas individually and explained the purpose, flow, expectations and outcomes. It was made clear to the managers at the outset that any efforts they had made to date to improve safety concerns would be recognized at the senior level. This new senior-level interaction at the frontline level was dealt with in a sensitive manner, so as not to be viewed as undermining the managers' operations of their units. The managers had an opportunity to debrief with the senior leader after the walkaround to clarify the progress made on any of the issues. Frontline staff was provided with a list of questions two days prior to the walkarounds. This allowed them to consider issues on their unit, and thus feel at ease when meeting with the senior leaders, which is a rare and potentially intimidating occurrence.

We also took measures to prepare the senior leaders. There was some initial apprehension amongst the senior leader group that not everyone had a clinical background and whether this would impact the effectiveness of the walkarounds. We reinforced the belief that in order for our organization to create and sustain a culture of safety, each and every senior leader had to demonstrate that they "walked the talk." We emphasized that leading a walkarounds session would be a visible and concrete method of accomplishing this, and would reinforce the message that everyone is accountable for patient safety. An information session was held with the senior leaders, during which the purpose and objectives were discussed, along with a script with suggested opening comments, questions to guide the conversation, and tips on how to redirect the dialogue if it were to divert to nonsafety matters.

Once the senior team clearly understands the mechanics of walkarounds, and the coordination of schedules is accomplished, the actual walkaround can be a dynamic and fruitful experience. When the participants are fully cognizant of the patient safety focus of this interaction, a skilled leader can elicit meaningful comments and suggestions for improvements. Good listening skills and constructive probing yield insights to the critical issues around patient safety on the units. The dialogue that develops over the space of an hour produces an opportunity to build a level of trust and understanding between the administrative arm of the organization and the clinical team. If the senior leader is able to effectively remove barriers and resolve issues identified by the participants, both the participants and the senior leader win. 
The senior leaders that have led walkaround sessions to date have been impressed with how dedicated caregivers are in providing a safe environment for patients. Amongst these leaders is a blend of those who have a clinical background and those that do not. It became clear at the outset that despite their background these senior leaders have an ability to use their expertise in understanding the clinical components of the issues and have an intuitive sense for the issues related to process and the barriers to providing safe care. Many examples of good communication and teamwork surface during these dialogues. As in any complex system, gaps in service delivery and communication are also fully evident. The interfaces between humans, their physical environment and advanced medical technology provide ample opportunities for improvements. Frontline staff see these gaps best.

By taking these steps to prepare all of the participants, we discovered that the senior leaders and the frontline staff were able to have a meaningful dialogue that was open and honest. There remains some apprehension among the managers; they are in a difficult position, in that their improvement efforts may be overshadowed by constraints at their level of management. We continue to work on this in order to alleviate any uneasiness and have found that providing an opportunity for the manager and senior leader to debrief at the end of walkarounds has yielded some success in addressing this issue. We feel confident that when well prepared, senior leaders do not require a clinical background in order to successfully conduct walkarounds. It appears that the remaining members of our senior leader team are keen to participate and connect with frontline staff, and thus make a contribution to safer patient care.

\section{BARRIERS AND ChAllenges}

Throughout the implementation of walkarounds, we have faced several barriers and challenges. One of the earliest challenges was the coordination of the various participants' schedules. It is important that walkarounds are conducted at a time that is convenient for the patient care area so that patient care is not compromised. However, coordinating the ebbs and flows of a busy patient care area and the hectic schedule of a senior leader proved to be more difficult than it appeared at the onset. Scheduling walkarounds with participants well in advance (3 to 6 months) may relieve some of these timing pressures.

Ensuring good communication and data flow is always a challenge, and contributes to the complexity of the walkarounds. There are five transfers of data within a short period of time, all requiring confirmation, prioritization and delegation (see Figure 1). We encountered some obstacles in ensuring the right personnel were contacted with enough information to move the process along. We imposed timelines in an effort to reduce bureaucratic delays. Making this flow of information seamless has proven to be an intricate task.
Another challenge was our ability to compile the data in a preexisting database. Spreadsheets and databases are required to store all comments, filter and sort data and create various reports. Given our organizational structure and need for communication at many different levels, it was necessary to develop methods of handling the data storage and communication needs.

\section{FINDINGS/LEARNINGS}

The data we collected from the first cycle of walkarounds has already demonstrated trends, the most significant being environmental gaps and aging facility infrastructures. This is consistent with other organizations that conduct walkarounds, and symptomatic of the more complex patient safety issues, such as teamwork and communication. We found that many times the environmental issues raised were longstanding and due to poor communication structures that remained unresolved. Participants' comments confirmed that organizations and services operating on multiple sites face challenges around communication and service delivery. Comments received from all of the sites indicate delays in obtaining various support services and equipment. It is important to view this as a systemic malfunction, as otherwise it may appear to be a non patient-safety-related issue. These environmental and equipment concerns may, in fact, have significant safety issues buried within. We anticipated that staff during their first encounter with senior leaders would raise environmental issues, which are less threatening and not as complex as clinical processes and safety-focused communication and teamwork.

It is our belief that initially staff are inclined to focus on basic environmental needs and issues that others are responsible for prior to addressing self-reflective professional practice related concerns. For example, various units expressed concerns relating to infection and prevention protocols, specifically focused on cleaning rooms, equipment, etc. With increasing volumes of patients (necessitating short turnaround times for cleaning rooms, beds and equipment), and the awareness of MRSA and other infectious diseases, staff need reassurance that appropriate monitoring and auditing of appropriate cleaning protocols is maintained. Consultations with both our IPC team and housekeeping services resulted in better clarity and communication about expectations and standards. We continue to improve the auditing processes that ensures staff and patients are protected and comfortable in isolation settings.

Beyond the trends in environmental gaps and aging facility infrastructures, we discovered a recurring level of dissatisfaction and opportunities for improvement around medication administration practices. The area designated for medication preparation and dispensing in one patient care area was not satisfactory compared to the unit-dose system that exists on all other patient care units. A unit-dose-based medication system has proven to 
Figure 1: Flow Schema for Patient Safety Walkarounds April 2005

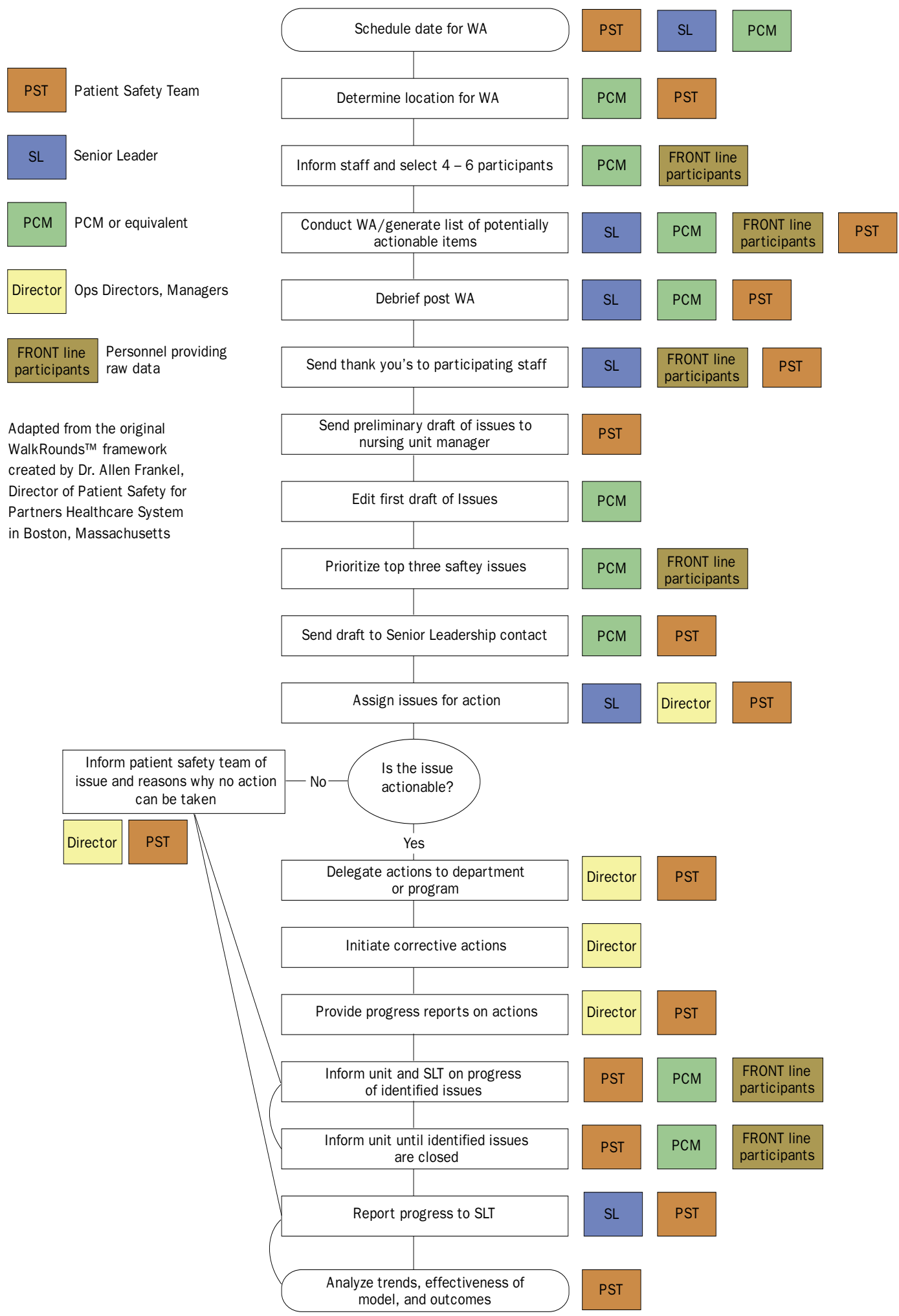


reduce the occurrence of medication errors. An assessment was completed and an application was submitted requesting the required updates. The application was approved and we are currently in the process of converting the medication delivery system in this patient care unit to a unitdose system.

Outside of the data that we have collected and the trends that have emerged, we have also learned many valuable lessons regarding the process and implementation of patient safety walkarounds. First and foremost, the development of trust between staff and senior leaders must be fostered and visible before a truly meaningful dialogue will occur during walkarounds. Despite the Accountability for Patient Safety policy and the Disclosure of Adverse Events policy, we recognized that it will take time for staff to feel safe in expressing their ideas and concerns on more difficult matters such as teamwork and communication. Until we reach this level, it is imperative that we continue to build trust by taking action and resolving the more cosmetic safety issues, such as broken equipment, in order to demonstrate the commitment of senior leadership to constructing a safer $S \& W$ healthcare organization.

Another lesson learned was that once a patient care area had selected their priority issues there remained many valuable comments and issues. In a few instances these issues were cause for concern amongst the Patient Safety Leadership Team, as they had the potential for negative outcomes. In order to avert such outcomes, the team decided to create a priority parking lot list where issues of concern are reviewed by the team on a monthly basis. If there were a consensus that an issue required immediate attention, the team would take the appropriate actions. All comments were then themed and compiled into a list to be reviewed by the senior leadership team. This provides the senior leaders with an overview of the ongoing trends.

One of the more encompassing lessons learned in implementing the walkarounds process is demanding in a large and

complex organization. Having a clear understanding of the multiple layers in an organization and who owns which piece of the puzzle is critical when it comes to delegating the issues for action. At the outset actions and information were flowing through several layers of individuals, which inevitably resulted in mixed messages and incomplete feedback. To streamline and avoid miscues, we reduced the number of individuals through which information flows, reducing confusion and creating a more fluid process. This is not to say that we have a seamless process; however, it is much improved, and will likely require further adjustments as we proceed and maneuver around future curves.

An organization must be
patient while developing
these new information flows,
as it will take some time
to reach truly meaningful
dialogues pertaining to
patient safety processes.

\section{RECOMMENDATIONS}

It is our firm belief that every healthcare organization has the ability to integrate patient safety walkarounds within their organization and have favourable outcomes. For any organization that is considering this type of program, we would make the following recommendations based on our experiences.

Another requirement for a successful implementation is to have a policy that defines the organization's commitment to patient safety, and articulates how staff is completely supported in reporting all near misses, safety hazards and adverse events. This policy must lay a foundation on which the conversation during walkarounds can be more open and honest, without staff fearing that they will be punished for sharing their experiences. During the intro- 
duction of each walkarounds, the senior leaders need to reiterate to the participants that their conversation will remain confidential, and to encourage them to speak openly and honestly about their experiences as care providers.

Walkarounds actively demonstrate the development of a culture of safety, where staff feel they are supported in patient safety initiatives, and where senior leaders are seen as partners in patient safety. When frontline staff and senior leaders have contracted to perform walkarounds, both will have invested significant resources to the process. To allow this focused energy to dissipate will imply to frontline staff that management is not deeply committed to patient safety at the bedside. Therefore, it is important to commit to a schedule, and provide replacement leaders should last-minute events otherwise cause a cancellation. It is imperative to understand that once this process takes root in the organization, walkarounds should not be discontinued, nor phased out. Once this happens it would become difficult to motivate staff to participate in any further patient safety initiatives.

Ensure that middle managers understand the focus and purpose of walkarounds, so that they do not feel undermined during this process. One needs to recognize the managers' efforts in resolving issues, and identify barriers that the manager has not been able to overcome. A debriefing after the rounds between the manager and the senior leader can reduce the amount of time a senior leader spends collecting background information. In time the senior leaders may be able to resolve systemic and communication blockages, provided they share their responses with other leaders.

An organization must be patient while developing these new information flows, as it will take some time to reach truly meaningful dialogues pertaining to patient safety processes. Be tolerant with resolving environmental and equipment issues: it is likely an organization will have to first address many such safety issues as bed brakes failing and showers flooding before tackling large systems issues such as communication processes during patient transfers. By addressing the environmental and equipment-related issues, the eventual yet essential trust between frontline staff and senior leadership takes shape. The corollary recommendation to the environmental issues is to prepare the support service staff for increased requests for services and better communication between service providers and units.

Further recommendations include agreeing on workable data management tools and communication strategies for identified priorities and improvements. Adapting communication tools to fit your organization's style and speed of response is important to ensure buy-in, but more importantly for staff to see results from this type of interaction. Also start with a small number of pilot units and engage the early adopters within the organization: doing so will provide critical feedback on what your organization requires in order to achieve success.

\section{CONCLUSION}

Walkarounds are used as a tool to unify the organization in solving systemic problems of communication and sharing common areas of concern. They are an excellent opportunity to address systemic patient safety issues in an effective project management /quality improvement framework. When conducted successfully, they serve to demonstrate the organization's commitment and accountability for safety in a very real, and visible, frontline manner.

\section{References}

Baker, R., Norton, P., Flintoft, V., Blais, R., Brown, A., Cox, J., Etchells, E., Ghali, W., Hebert, P., Majumdar, S., O'Beirne, M., Palacios-Derflingher, L., Reid, R., Sheps, S., Tamblyn, R. 2004. "The Canadian Adverse Events Study: the incidence of adverse events among hospital patients in Canada." Canadian Medical Association Journal. 170 (11): $1678-1686$.

Frankel, A., Graydon-Baker, E., Neppl, C., Simmonds, T., Gustafson, M., Gandhi, T. 2003. "Patient Safety Leadership WalkRounds." Joint Commission Journal on Quality and Safety. 29(1): 16-26.

\section{About the Authors}

Guna Budrevics, C.C.H.S.A.(A), CPHQ, Coordinator, Quality and Patient Safety, Sunnybrook and Women's College Health Sciences Centre has led numerous quality improvement and patient safety initiatives at Sunnybrook and Women's College Health Sciences Centre.

Catherine O'Neill, RN, Patient Safety Specialist, Sunnybrook and Women's College Health Sciences Centre co-founded the Patient Safety Service at Sunnybrook and Women's Health Sciences Centre in July 2002. The Patient Safety Service is dedicated to identifying, understanding, and reducing errors related to patient care, as well as, promoting a culture of safety through leadership, education and support for continuous improvement to all staff within our organization.

Corresponding Author: Guna Budrevics, Sunnybrook and Women's College Health Sciences Centre, 2075 Bayview Avenue, Toronto, Ontario M4N 3M5. Email: guna.budrevics@sw.ca Tel: 416-480-5918 